\title{
Rabdomiólisis intraoperatoria en cirugía de resección de osteosarcoma de fémur: Reporte de caso y discusión
}

\author{
Case report: Intraoperative rhabdomyolisis after femur \\ osteosarcoma resection
}

Luis Pablo Masco', Silvina Longo'

\begin{abstract}
Rhabdomyolysis is a pathology that rarely has causes in the perioperative period, where it has been commonly related as a complication of malignant hyperthermia, prolonged patient positioning with intraoperative muscle compression, in the postoperative period of bariatric surgery and in children. The purpose of this review is to present the case of a 49 year-old male patient, who underwent limb salvage surgery for treatment of a left femur osteosarcoma, with reconstruction via bone transplant and joint prosthesis. During the procedure hyperkalemia and elevation of Creatine-Phosphokinase (CPK) enzyme levels where detected, without changes compatible with renal failure, which required repeated treatment to normalize and that, after ruling out other causes, it was attributed to skeletal muscle destruction during the procedure. Rhabdomyolysis is a phenomenon inherent to this sort of procedures and may manifest initially as laboratory findings and that, if not diagnosed in time, may lead to fatal arrhythmias and acute renal failure.
\end{abstract}

\section{RESUMEN}

La rabdomiólisis es una patología que rara vez tiene origen en el período perioperatorio, donde comúnmente se le ha relacionado como complicación de la hipertermia maligna, de decúbitos prolongados con compresión muscular intraoperatoria, del posoperatorio de la cirugía bariátrica y en niños. El objetivo de este trabajo es presentar el caso de un hombre de 49 años, sometido a resección de un osteosarcoma de fémur izquierdo con reconstrucción mediante trasplante

\section{Key words:}

Rhabdomyolysis, renal injury, orthopedic surgery

\section{Palabras clave:}

Rabdomiólisis,

fallo renal, cirugía ortopédica

Servicio de Anestesiología, Hospital Privado Universitario de Córdoba, Argentina.

Fecha de recepción: 27 de abril de 2018

Fecha de aceptación: 30 de mayo de 2018

\section{ORCID}

https://orcid.org/0000-0003-3746-6229

Correspondencia:

Dr. Luis Pablo Masco

Email: luismasco@gmail.com 
óseo y prótesis articular, durante el cual se detectan hiperpotasemia y aumento de la Creatin-Fosfokinasa (CPK), sin alteraciones compatibles con fallo renal, que requirió reiterados tratamientos para la normalización de los valores de kalemia, y que tras descartar otras causas se atribuyó a la destrucción de musculoesquelético durante el procedimiento. La rabdomiólisis es un fenómeno inherente a este tipo de procedimientos y puede manifestarse inicialmente con alteraciones analíticas que, de no ser diagnosticadas en tiempo y forma, pueden llevar a arritmias fatales y fallo renal agudo.

\section{Reporte de Caso}

C omunicamos el caso de un hombre de 49 años, caucásico, guardabosques de profesión, peso $100 \mathrm{~kg}$, estatura $1,87 \mathrm{~m}$, con actividad física regular, con antecedente de hipotiroidismo en tratamiento y control, que sufre caída de propia altura tras lo cual queda con dolor persistente en el muslo izquierdo, que se fue intensificando a lo largo de un año. Por lo anterior, se le realizan estudios de imágenes que identifican lesiones en diáfisis proximal de fémur izquierdo por lo que se programa biopsia. En los días previos a la intervención sufre caída de propia altura presentando una fractura en hueso patológico del fémur izquierdo. Tres meses después de la reagudización se llega al diagnóstico de osteosarcoma fibroelástico grado III en fémur izquierdo.

El paciente recibe seis ciclos de quimioterapia neoadyuvante (durante la cual el paciente sufre fallo renal agudo que revierte casi en su totalidad, quedando con deterioro leve de la función renal) y se programa resección quirúrgica con reconstrucción mediante trasplante óseo y prótesis de cadera, por lo cual el paciente fue evaluado por el Servicio de Cardiología y se realizaron estudios prequirúrgicos que resultaron normales (Tabla 1).

Se realizó anestesia general y epidural (para analgesia intra y posoperatoria), se colocó catéter central en vena yugular interna derecha (para infusión de drogas, hidratación y control de presión venosa central) y catéter en arteria radial izquierda para monitoreo invasivo de la presión arterial. El procedimiento tuvo una duración de 10 horas, durante el cual se detectó hiperkalemia de 5,2 mEq/L y luego de 5,4 mEq/L (Figura 1), por lo cual se decide comenzar con maniobras de redistribución de potasio (gluconato de calcio EV $1.000 \mathrm{mg}$ y salbutamol vía inhalatoria $400 \mathrm{mcg}$ ). En nuevo control se constata persistencia y aumento de hiperkalemia $(5,6 \mathrm{mEq} / \mathrm{L})$ y se realizan nuevas maniobras (Insulina corriente $10 \mathrm{UI}$ diluida en $500 \mathrm{ml}$ de dextrosa $10 \%$ en agua, y $100 \mathrm{mEq}$ de bicarbonato de sodio) con lo que se logra normalizar los valores.

En la sala de recuperación postanestésica (SRPA), un nuevo control revela hiperpotasemia, por lo cual se repite tratamiento con bicarbonato de sodio e insulina.

Durante el procedimiento el paciente tuvo una diuresis promedio de $5 \mathrm{ml} / \mathrm{kg} / \mathrm{h}$, sin mioglobulinuria macroscópica. La uremia y creatininemia se mantuvieron en valores habituales durante toda la internación, manifestados por un clearence de creatinina estable.

La enzima CPK demostró elevación leve durante el procedimiento (llegando a $857 \mathrm{U} / \mathrm{L}$ en el intraoperatorio) y luego se constató aumento progresivo con pico a las 24 h (5.305 U/L) y tendencia a la normalización hacia el $5^{\text {to }}$ día posoperatorio. Los siguientes controles mostraron continua corrección de todos los valores de laboratorio.

El paciente permaneció internado por tres días en

\begin{tabular}{lcc}
\multicolumn{3}{c}{ Tabla 1. Valores de laboratorio prequirúrgico } \\
\hline Examen & Valor & Unidad \\
Hemoglobina & 10,5 & $\mathrm{~g} / \mathrm{dL}$ \\
Hematocrito & 30,1 & $\%$ \\
Sodio & 138 & $\mathrm{mMol} / \mathrm{L}$ \\
Potasio & 4,7 & $\mathrm{mMol} / \mathrm{L}$ \\
Cloro & 101 & $\mathrm{mMol} / \mathrm{L}$ \\
Fósforo & 4,1 & $\mathrm{mg} / \mathrm{dL}$ \\
Calcio & 9,9 & $\mathrm{mg} / \mathrm{dL}$ \\
Clearence de creatinina & 60 & $\mathrm{ml} / \mathrm{min}$ \\
Lactacidemia & 1,1 & $\mathrm{mMol} / \mathrm{L}$ \\
\hline
\end{tabular}

* Las horas de cirugía se expresan desde el inicio de la anestesia (p. ej. H2 son dos horas desde el comienzo. +1, +2, etc. Se refieren a días de internación posprocedimiento. Abreviaturas: PR: Prequirúrgico; S.R.P.A.: Sala de Recuperación Posanestésica. 


\section{Tabla 2. Alteraciones analíticas más frecuentes en rabdomiólisis}

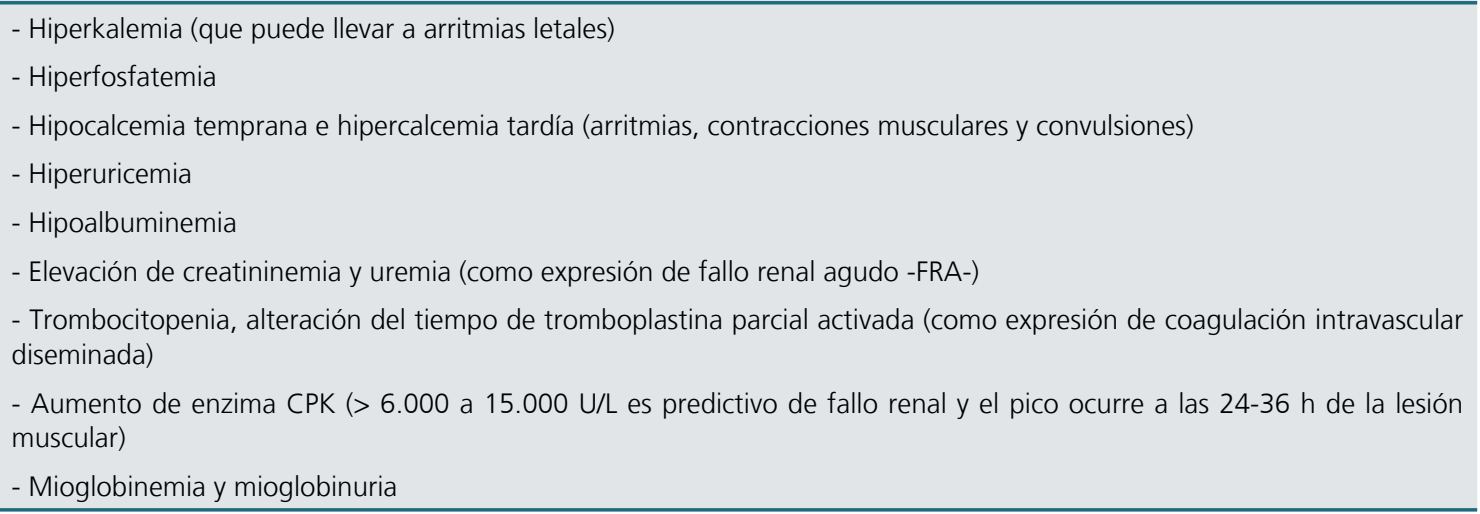

la Unidad de Cuidados Intermedios, y luego en sala de intervención común donde evolucionó favorablemente y fue dado de alta en el séptimo día del posoperatorio.

\section{Discusión}

La rabdomiólisis es un fenómeno patológico caracterizado por la destrucción de células de músculo esquelético, que lleva a la liberación de componentes intracelulares (principalmente potasio, fósforo, tratos, CPK y mioglobina) al torrente sanguíneo que pueden ser detectadas mediante análisis sanguíneo y urinario y que pueden conducir a severos trastornos, como arritmias cardíacas por alteraciones electrolíticas y lesión renal aguda por nefrotoxicidad secundaria al exceso de mioglobina.

Sus principales etiologías se pueden dividir en hipóxicas, físicas, químicas, biológicas e idiopáticas[11], siendo las causas más comunes en el adulto las drogas ilegales, abuso de alcohol, medicamentos, distrofias musculares, trauma, convulsiones, postración y el síndrome neuroléptico maligno[12]. La causa quirúrgica por manipulación, disección o resección muscular en el transcurso de un procedimiento es poco o no mencionada, y quedaría incluida en la etiología física.

La presentación clásica ( $50 \%$ ) en el paciente consciente está dada por la tríada de: mialgias, debilidad generalizada y orina oscura. Los síntomas no específicos incluyen fiebre, palpitaciones, náuseas y vómitos. Generalmente, la anamnesis directa o indirecta revela un agente causal.

Este síndrome, así como los datos provenientes del examen físico, son poco útiles en el diagnóstico en un paciente bajo sedación/anestesia general, excepto por la hipertermia y diuresis oscura en un paciente con sondaje vesical, lo que obliga al diagnóstico mediante una alta sospecha clínica en base al tipo de procedimiento, el monitoreo y los análisis de laboratorio intra y posoperatorios. En la Tabla 2, podemos observar las alteraciones analíticas más frecuentes incluyen.

El tratamiento está dirigido a corregir las alteraciones de laboratorio, antes que el paciente se encuentre en una situación de riesgo vital:

- Monitoreo (control de signos vitales básicos, diuresis, electrocardiografía).

- Fluidoterapia agresiva.

- Corrección de alteraciones electrolíticas (principalmente Hiperkalemia y/o hiperfosfatemia), del estado ácido/base y metabólicas.

- Prevención/tratamiento de FRA (sobre todo según factores de riesgo: Pico de CPK mayor a 6.000 $\mathrm{UI} / \mathrm{L})$.

- Tratamiento de deshidratación (expresada analíticamente como hematocrito $>50 \%$, sodio sérico $>150 \mathrm{mEq} / \mathrm{L}$, ortostatismo, presión de enclavamiento pulmonar $<5 \mathrm{~mm} \mathrm{Hg}$, fracción excretada de sodio en orina $<1 \%$ ).

- Tratamiento de sepsis e hipoalbuminemia.

Una vez que se sospecha y diagnostica la rabdomiólisis se debe aumentar la administración de líquidos y lograr una diuresis forzada, inclusive recurriendo a diuréticos, medidas para evitar consecuencias fatales, dado que los niveles de CPK reflejan la severidad de la lisis de las células musculares y se ha relacionado con mayor riesgo de desarrollar insuficiencia renal aguda. Por esta razón es necesaria su medición temprana en pacientes de riesgo para iniciar una terapéutica eficaz.

La cirugía oncológica de resección de osteosarcoma con intención de evitar la amputación del miem- 
bro afectado es considerada actualmente el procedimiento de elección en el tratamiento de esta patología[14],[15], dado su pico de incidencia en pacientes en la segunda década de vida y la notable mejoría de sobrevida con técnicas combinadas de quimioterapia y cirugía[13].

La manipulación, disección, resección, compresión e isquemia del tejido muscular es un hecho inherente al procedimiento, y produce rabdomiólisis en distintos grados, incluso sin existir momentos de clampeo arterial y el riesgo de lesión por isquemia/ reperfusión.

Por eso consideramos que el reconocimiento temprano de esta complicación puede mejorar la morbimortalidad en estos pacientes, que suelen ser jóvenes y sin comorbilidades de importancia, y es algo que el equipo quirúrgico y sobre todo el anestesiólogo a cargo deben tener presente.

\section{Referencias}

1. Miller R, Eriksson L, Fleisher $\mathrm{L}$, Wiener-Kronish J, Cohen N, Young W. Miller's Anesthesia (8th Edition).

2. Wappler $F$, Fiege $M$, Steinfath M, Agarwal K, Scholz J, Singh S, et al. Evidence for susceptibility to malignant hyperthermia in patients with exercise-induced rhabdomyolysis. Anesthesiology. 2001 Jan;94(1):95-100. https:// doi.org/10.1097/00000542200101000-00019

PMID:11135728

3. Bertrand $M$, Godet $G$, Fléron $\mathrm{MH}$, Bernard MA, Orcel P, Riou $B$, et al. Lumbar muscle rhabdomyolysis after abdominal aortic surgery. Anesth Analg. 1997 Jul;85(1):11-5. PMID:9212115

4. Gurnaney H, Brown A, Litman RS. Malignant hyperthermia and muscular dystrophies. Anesth Analg. 2009 Oct;109(4):10438. https://doi.org/10.1213/ ane.0b013e3181aa5cf6 PMID:19762730

5. Mathes DD, Assimos DG, Donofrio PD. Rhabdomyolysis and myonecrosis in a patient in the lateral decubitus position. Anesthesiology. 1996 Mar;84(3):727-9. https:// doi.org/10.1097/00000542199603000-00030 PMID:8659803

6. Lagandré S, Arnalsteen L, Va-
Ilet B, Robin E, Jany T, Onraed $B$, et al. Predictive factors for rhabdomyolysis after bariatric surgery. Obes Surg. 2006 Oct;16(10):1365-70. https:// doi.1381/096089206778663643 PMID:17059748

7. Faintuch J, de Cleva R, Pajecki D, Garrido AB Jr, Cecconello I. Rhabdomyolysis after gastric bypass: severity and outcome patterns. Obes Surg. 2006 Sep;16(9):1209-13. https:// doi.1381/096089206778392202 PMID:16989706

8. Gelbart B, DeMarco R, David Hussey A, Namachivayam SP, McRae R, Quinlan C, et al. Rhabdomyolysis in a Tertiary PICU. Pediatric Critical Care Medicine [Internet]. Ovid Technologies (Wolters Kluwer Health); 2018 Jan;19(1):e51e57. http://dx.doi.org/10.1097/ pcc.0000000000001397

9. Elsayed EF, Reilly RF. Rhabdomyolysis: a review, with emphasis on the pediatric population. Pediatr Nephrol. 2010 Jan;25(1):7-18. https://doi. org/10.1007/s00467-009-12239 PMID:19529963

10. Luck RP, Verbin S. Rhabdomyolysis: a review of clinical presentation, etiology, diagnosis, and management. Pediatr Emerg Care. 2008 Apr;24(4):2628. https://doi.org/10.1097/ PEC.0b013e31816bc7b7
PMID:18418269

11. Domínguez Jl, Campero JM, Reyes M, San Martín MF. Caso clínico: Rabdomiólisis secundaria al consumo de cocaína. Revista Chilena de Anestesia [Internet]. Asociación de Médicos Anestesiólogos de Chile; 2018 Apr 5;47(1). http://dx.doi. org/10.25237/revchilanestv47n01.05

12. Khan FY. Rhabdomyolysis: a review of the literature. Neth J Med. 2009 Oct;67(9):272-83. PMID:19841484

13. Misaghi A, Goldin A, Awad M, Kulidjian AA. Osteosarcoma: a comprehensive review. 2018;4:12. Société Internationale de Chirurgie Orthopédique et de Traumatologie Journal, 4, 12. https://doi.org/10.1051/sicotj/2017028.

14. Yang $Y$, Han L, He Z, Li X, Yang S, Yang J, et al. Advances in limb salvage treatment of osteosarcoma. Journal of Bone Oncology [Internet]. Elsevier BV; 2018 Mar;10:36-40. http://dx.doi.org/10.1016/j. jbo.2017.11.005

15. Brown HK, Schiavone K, Gouin F, Heymann MF, Heymann D. Biology of Bone Sarcomas and New Therapeutic Developments. Calcif Tissue Int. 2018 Feb;102(2):174-95. https://doi. org/10.1007/s00223-017-03722 PMID:29238848 